\title{
Performance Analysis of WiMAX Network with Uplink Scheduling Algorithm and its Implementation
}

\author{
Stuti Upadhyaya ${ }^{1}$, Deepak Bicholia ${ }^{2}$ \\ Research Scholar, Dept. of Electronics \& Communication Engineering, Institute of Engineering \& Science, \\ IPS Academy, Indore (M.P.) India ${ }^{1}$ \\ Asst. Professor, Dept. of Electronics \& Communication Engineering, Institute of Engineering \& Science, \\ IPS Academy, Indore (M.P.) India ${ }^{2}$
}

\begin{abstract}
The IEEE 802.16 provides QoS support, but it does not specify the details of how the QoS can be achieved for different types of applications. Since the gradual deployment of new multimedia applications generate traffic that is more sensitive to delay thus it required proper scheduling of packets. The objective of this paper is to analysis WiMAX network with and without scheduling methods by varying nodes density from 2 to 10 nodes. To recapitulate, WiMAX 802.16d gives better results with scheduling method (round robin) which is almost same with time and the performance parameters are throughput, packet delivery ratio and end-to-end delay.
\end{abstract}

Keywords: IEEE 802.16, Uplink Scheduling, WiMAX, QoS, throughput, packet delivery ratio, end-to-end delay.

\section{INTRODUCTION}

A WiMax network has a number of initial base stations and associated antennas communicating by wireless to a much larger number of client devices a point to multipoint configuration. Base stations are either directly wired to the Internet or use WiMax links to other base stations that are so connected. Client devices initially are generally small, building - mounted antenna/transreceiver systems to which in building LANs (such as WLANs) are connected. But future clients - depending on the frequency bands used will often integrate into end user devices, such as notebook PCs and, eventually, mobile devices, such as smart phones. Each base station provides wireless coverage over an area called a cell. [2]

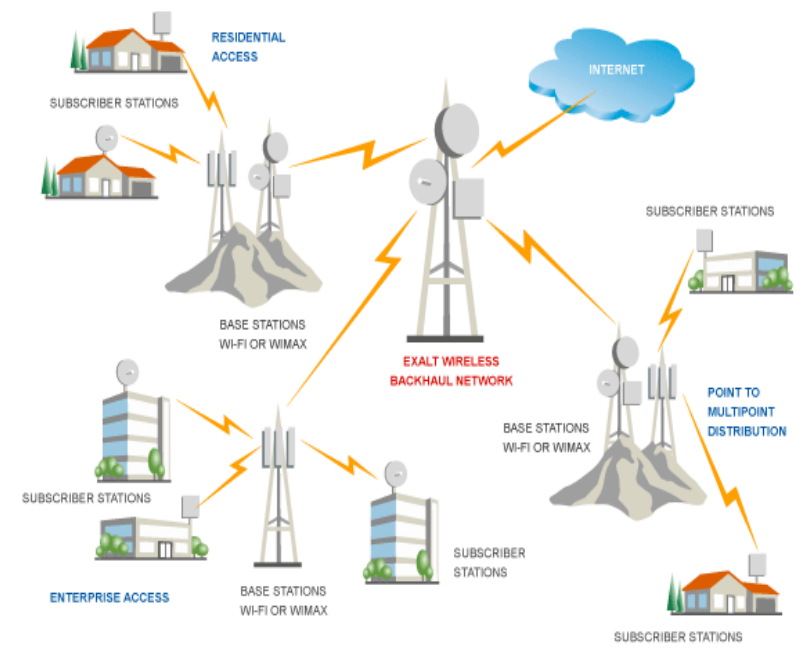

Figure: WiMAX Network
A WiMAX system consists of two parts:

1) WiMAX Base Station Tower: A single WiMAX tower can provide coverage to a very large area - as big as 3,000 square miles $(\sim 8,000$ square $\mathrm{km})$.

2) WiMAX Receiver: The receiver and antenna could be a small box or PCMCIA card, or they could be built into a laptop the way WiFi access is today. [1]

\section{WiMAX Parameters}

\begin{tabular}{|l|l|}
\hline \multicolumn{1}{|c|}{ Frequency } & $2-11 \mathrm{GHz}$ \\
\hline Speed & $30 \mathrm{mbps}$ \\
\hline Modulation & BPSK,QPSK, 16-QAM, \\
Technique & 64-QAM \\
\hline FFT Size & 256 \\
\hline Bit Rate & $5 \mathrm{bps} / \mathrm{Hz}$ \\
\hline Channel Bandwidth & $1.25-20 \mathrm{MHz}$ \\
\hline Coding & $\begin{array}{l}\text { Convolution, Turbo, RS } \\
\text { Encoder }\end{array}$ \\
\hline
\end{tabular}

\section{PROBLEM STATEMENT}

A WiMAX network is designed to incorporate different types of data streams, and it aims to provide guarantee of quality of services for all the data streams being served by WiMAX. The IEEE 802.16 standard provides specification for WiMAX MAC layer. In the MAC layer, one challenge is the diversified service, which requires the scheduling scheme to be adaptive for the various QoS parameters of different service types. When we analyzed 
WiMAX network, we observed that there is lot of fluctuations in packet delivery ratio, throughput and specially end-to-end delay. The reason behind this was packet drop and packet collision because of lack of scheduling. To improve this we have implemented the simplest scheduling algorithm i.e. Round Robin Scheduling. WiMAX standard defines adequate service classes to support scheduling, but does not define the algorithms for them. This absence of definition allows more flexibility in the implementation of scheduling algorithm. The research problem being investigated here is, after connections are admitted into the WiMAX network, how to allocate scheduling services, so that the QoS requirements of the connections can be satisfied.

\section{PROTOCOL LAYERS OF WIMAX}

The IEEE 802.16 standard applies the OSI model. The 802.16 standard defines only the two lowest layers, the PHYsical Layer and the MAC Layer. The MAC layer is itself made of three sublayers, the CS (Convergence Sublayer), the CPS (Common Part Sublayer) and the Security Sublayer. [2]

These sub layers are discussed below:

1. Convergence Sublayer (CS): The CS is just above the MAC CPS sublayer. The CS accepts higher-layer PDUs and classifies them into appropriate QoS Classes.

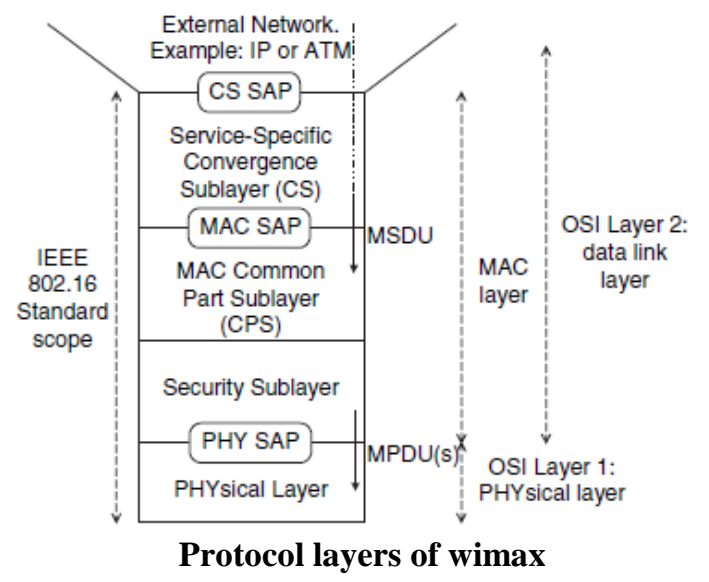

2. Medium Access Control Common Part Sublayer (MAC CPS):

The Common Part Sublayer (CPS) resides in the middle of the MAC layer. The CPS represents the core of the MAC protocol and is responsible for:

- bandwidth allocation;

- connection establishment;

- Maintenance of the connection between the two sides.

\section{Security Sublayer:}

The MAC Sublayer also contains a separate Security Sublayer providing authentication, secure key exchange, encryption and integrity control across the BWA system. [3]

\section{III.WIMAX QOS ARCHITECTURE}

The figure below gives an illustration of the QOS architecture as specified in the IEEE 802.16 standard. The MAC part of IEEE 802.16 is connection oriented. After the basic process of network initialization, each SS establishes a connection with the WiMAX BS. Each connection of a SS is assigned a unique connection identifier (CID) by the WiMAX BS. In subsequent communication between the BS and the SS, the SS has to use this CID. A service flow is a set of packets that is promised a particular QoS. Each service flow is assigned a service flow identifier (SFID). A service flow is usually defined for a user depending on his service requirements. A connection is mapped to a service flow through a SFID. There is a bandwidth scheduling module at both the MS and the BS. This module is responsible for allocating the bandwidth, among different connections that are present at the SS and between different SSs requests at the WiMAX BS. [4]

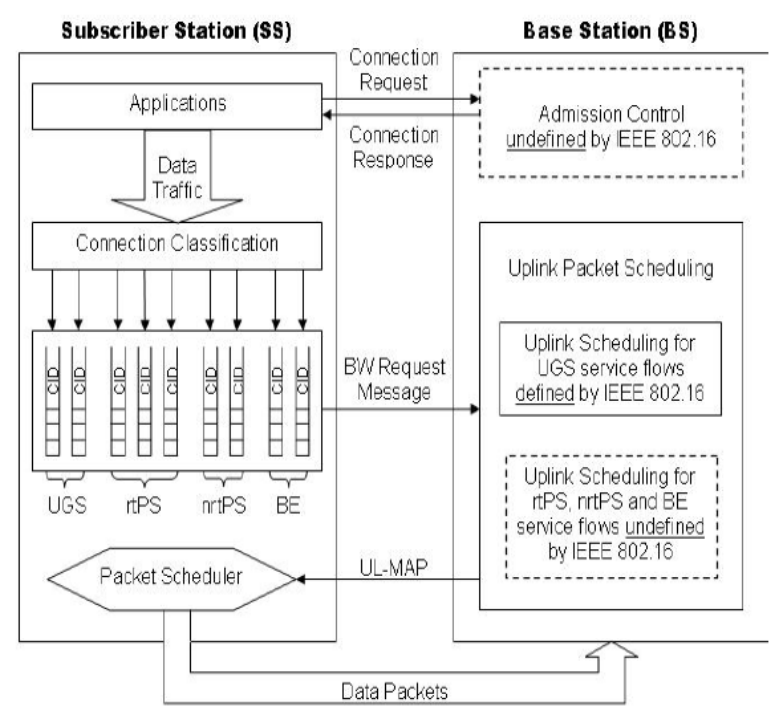

Figure: QoS Architecture

\section{SCHEDULING}

The most important feature of 802.16 is providing support for QOS. It means that each packet is associated with a particular service flow to meet its requirements. Each service flow is assigned a set of QOS parameters accordingly. Depending on the application requirement, different applications can request for different service flows. A service class has to be defined and the service flow is associated to it. To support different applications 802.16 has specified five scheduling services: UGS, rtPS, ertps, nrtPS and BE. Each scheduling service is associated with a service class. [5]

The QoS requirements of different applications are met by mapping the requirements into certain bandwidth allocation. The scheduling classification is specified by the standard based on the type of application. The five defined QoS classes are described as follows: 
Unsolicited Grant Service (UGS) supports service flows that generate fixed size data packets in real-time at regular intervals. The BS on its own provides fixed bandwidth allocation at regular intervals.[6]

Real-Time Polling Service (rtps) supports service flows that generate variable size data packets in real-time on a regular basis. In this case, each SS specifies the amount of bandwidth it needs. [7]

Extended Real-time Polling Service (ertps) supports the need of applications that have real-time service flows. In ertps the grants are not fixed. SS can send a request to change bandwidth size. [8]

Non-Real-Time Polling Service (nrtPS) supports the applications that generate variable size data packets. The bandwidth request can be sent by SS using the contention based request or SS can send a request. [9]

Best Effort (BE) supports the requirements of applications that do not require any guarantees on throughput or delay. The SS can use contention based request for requesting bandwidth. [10]

\section{SCHEDULING ALGORITHM}

The wimax standard provides specification for different services, but does not specify any scheduling algorithm. The scheduling algorithm used here is:

\section{Round Robin (RR):}

Round Robin as a scheduling algorithm is the most basic and least complex scheduling algorithm. It has a complexity value of $\mathrm{O}$ (1). Basically the algorithm services the backlogged queues in a round robin fashion. Each time the scheduler pointer stop at a particular queue, one packet is dequeued from that queue and then the scheduler pointer goes to the next queue. This is shown in Figure. It distributes channel resources to all the SSs without any priority. The RR scheduler is simple and easy to implement. However, this technique is not suitable for systems with different levels of priority and systems with strongly varying sizes of traffic. So, the procedure of RR scheduler works in rounds by serving the first packet in each priority queue in sequence according to their precedence till all queues are served and then it restarts over to the second packet in each queue.[11]

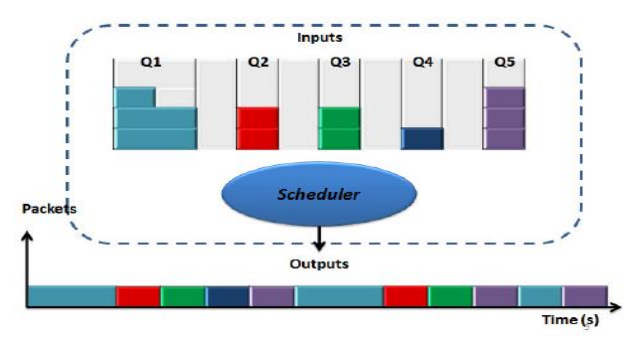

Figure: Round Robin Scheduler

\section{VII.SIMULATION SCENARIO}

The simulation tool used is NS2 with WiMAX implementation from NIST. The current WiMAX implementation has OFDMA phy with time division duplexing mechanism (TDD), fragmentation and reassembly of frames, service flow and QOs scheduling, CDMA based contention resolution. The scheduler implemented is a round robin scheduler that provides uplink allocation to all subscriber station based on the bandwidth requested by each SS.

\section{Simulation Parameters}

\begin{tabular}{|c|c|}
\hline Simulation Tool & NS-2.35 \\
\hline IEEE Scenario & 802.16 \\
\hline Propagation & Two Ray Ground \\
\hline Number of nodes & 2 to 10 nodes \\
\hline Traffic Type & TCP \\
\hline Antenna & Omni directional \\
\hline MAC Type & IEEE 802.16 \\
\hline Routing Protocol & AODV \\
\hline Queue limit & 50 Packets \\
\hline Simulation area & 2 KM \\
\hline Queue type & Droptail, CMU priqueue \\
\hline Channel & Wireless Channel \\
\hline Simulation time & 20 sec. \\
\hline Scheduling Algorithm & Round Robin \\
\hline
\end{tabular}

\section{Evaluation of Results:}

I have implemented a WiMAX Scenario in NS-2 with and without scheduling process with varying node density from 2-10 nodes for 20sec simulation time. The scheduler implemented is a round robin scheduler. The results are analyzed with the use of various performance matrices like Packet Delivery Ratio, End to End delay and overall Throughput.

\section{Packet Delivery Ratio}

Figure shows the Packet delivery ratio with scheduling and without scheduling for 2 nodes to 10 nodes. The graph is plotted between $\mathrm{x}$-axis i.e. number of nodes and $\mathrm{y}$-axis i.e. packet delivery ratio.

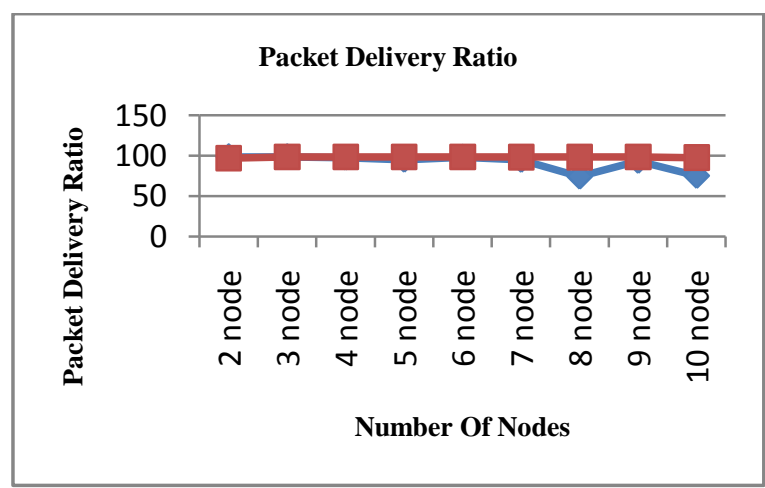

Figure: Packet Delivery Ratio comparison with and without scheduler 
The blue line indicates PDR without scheduler and red line indicates PDR with scheduler.

Analysis of Packet Delivery Ratio: From the above figure we analyzed that with scheduling we have constant Packet Delivery Ratio in comparison of without scheduling for different node density scenario which varying from 2 to 10 nodes which means that the data loss using scheduling is less.

\section{Throughput}

Figure shows the throughput with scheduling and without scheduling from 2 nodes to 10 nodes. The graph is plotted between $\mathrm{x}$-axis i.e. number of nodes and $\mathrm{y}$ - axis i.e. throughput. The blue line indicates throughput without scheduler and red line indicates throughput with scheduler.

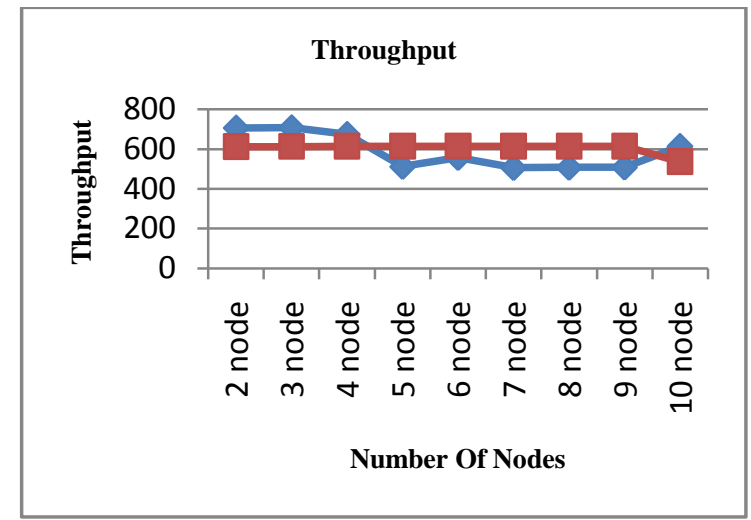

Figure: Overall Throughput with \& without scheduling

Analysis of Overall Throughput: From the above figure we analyzed that the throughput with scheduling is constant in comparison of without scheduling for different node density scenario which varying from 2 to 10 nodes, which means there is less packet drop.

\section{End to End Delay}

Figure shows the End to End Delay with scheduling \& without scheduling for 2 nodes to 10 nodes. The graph is plotted between $\mathrm{x}$-axis i.e. number of nodes and $\mathrm{y}$ - axis i.e. end-to-end delay. The blue line indicates end-to-end delay without scheduler and red line indicates end-to-end delay with scheduler.

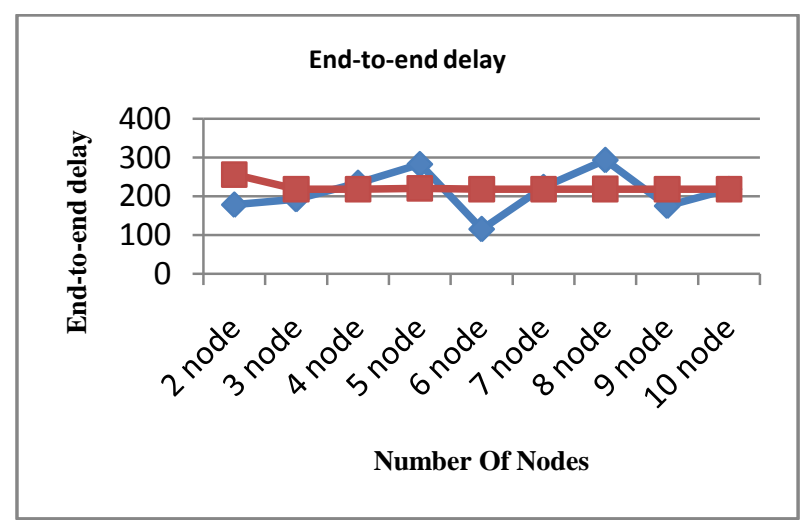

Figure: End to End Delay with \& without scheduling
Analysis of End to End Delay: From the above figure we analyzed that the End to End Delay remains constant with scheduling in comparison of without scheduling for different node density scenario which varying from 2 to 10 nodes.

\section{CONCLUSION}

In this work we analyzed parameter like Packet Delivery Ratio, End to End Delay and Overall Throughput and concluded that the WiMAX scenario without scheduling fluctuates a lot rather than WiMAX scenario with scheduling, which remains constant all the time for each traffic type i.e. 2 nodes, 3 nodes, 4 nodes, 5 nodes, 6 nodes, 7 nodes, 8 nodes, 9 nodes and 10 nodes with $20 \mathrm{sec}$ simulation time for two ray ground propagation in IEEE 802.16 scenario for omni directional antenna.

\section{REFERENCES}

[1]. Upen Dalal, Wireless communication, Oxford University Press, Second Edition.

[2]. Sanida Omerovic, "WiMax Overview", Faculty of Electrical Engineering, University of Ljubljana, Slovenia.

[3]. Loutfi Nuaymi, WiMAX technology for broadband wireless access, John wiley \& sons, Ltd.

[4]. Saurabh Dubey and Sachin Kumar, "Security Issues in WiMAX: A Critical Review", International Journal of Information and Computation Technology, Volume 3, Number 3 (2013), pp. 189194.

[5]. Kalpalatha Sankarasubramaniam, Srikanth Subramanian, "A Performance Study of Uplink Scheduling in WiMAX Network", IEEE , 2012.

[6]. Alrahman Mohamed Abdalla, Dr. Amin Babiker A Nabi, Dr. Ashraf A. Osman, "A QoS Oriented Analysis of ertPS and UGS flows in voice application over WIMAX" , International Journal of Recent Trends in Engineering \& Research (IJRTER) Volume 02, Issue 08; August - 2016.

[7]. Sunita Kumari, Virender Sharma, "A Survey on conventional/Turbo code over IEEE 802.16 based Network", SSRG International Journal of Computer Science and Engineering (SSRGIJCSE) - EFES April 2015.

[8]. Mohit Chhabra, Vikrant Manocha, Shanu Malhotra, "A Survey on adaptive scheduling and digital modulation schemes over IEEE 802.16 Standard", IJARIIE Vol-2 Issue-4 2016.

[9]. Ramjee Prasad, Fernando J. Velez, "WiMAX Networks: TechnoEconomic Vision and Challenges", springer science+buisness media B.V. 2010.

[10]. Yong Zhang, Chao Dai, Mei Song, "A Novel QoS Guarantee Mechanism In IEEE 802.16 Mesh Networks", Computing and Informatics, Vol. 29, 2010.

[11]. Harpreet Kaur, Gurpal Singh, "Implementation and Evaluation of Scheduling Algorithms in Point-to-Multipoint Mode in Wimax Networks", IJCST Vol. 2, Issue 3, September 2011. 